\begin{tabular}{|l|l|l|l|}
\hline Eiszeitalter u. Gegenwart & 37 & $\begin{array}{l}79-91 \\
5 \mathrm{Abb} .\end{array}$ & Hannover 1987 \\
\hline
\end{tabular}

\title{
Der kaltzeitliche Formenschatz im Cleurie-Tal (Südwestvogesen)
}

\author{
GERD WENZENS *) \\ Pleistocene (3 glacial periods), Würm glaciation, moraines, fluvioglacial features, \\ fluviolacustrine features, extent of glaciers \\ Vosges, Cleurie Valley
}

Kurzfassung: Kartierung und Aufschlußanalyse der glazialen und fluvioglazialen Formen und Sedimente im CleurieTal lassen folgende Aussagen zur Vergletscherungsgeschichte dieses Raumes zu:

Anhand der Kriterien Erhaltungszustand, Verwitterungsintensität bzw. Verfestigungsgrad, Höhenlage und Zusammensetzung können glaziale Ablagerungen aus drei verschiedenen Kaltzeiten nachgewiesen werden.

Aufgrund der Eismächtigkeiten des Mosel- und MoselotteGletschers im Becken von Remiremont/St. Amé kann im Würm eine Moselotte-Diffluenzzunge allenfalls bis in das Gebiet südlich von le Tholy gereicht haben. Dies wird durch die maximale Höhenlage der Würmsedimente östlich von le Tholy bestätigt: Sie lassen als Ablagerungsmedium lediglich den Vologne-Gletscher sowie kleinere Seitengletscher zu. Die damalige Schneegrenze lag bei ca. $850 \mathrm{~m}$.

Die Wälle östlich von le Tholy sind fluvioglazialer bzw. lakustrischer Entstehung. Schmelzwässer aus dem Cellet-Tal wurden vom Vologne-Gletscher gestaut, so daß sich hier mächtige Deltasedimente ablagern konnten. Während einer Abschmelzphase des Vologne-Gletschers bildete sich hinter der Endmoräne von Bas Beillard das Delta von le Costet, dessen glazio-lakustrische Ablagerungen von proglazialen Sedimenten bedeckt wurden, die im Zusammenhang mit der Ausbildung der Endmoräne von Pré Chaussote entstanden.

\section{[Pleistocene Morphology of the Cleurie-Valley} (Southwestern Vosges)]

Abstract: Mapping and profile analysis of glacial and fluvioglacial forms and deposits in the Cleurie valley allow the following statements concerning this area's glacial history:

On basis of criterions like state of conservation, intensity of weathering and incrustation, height and composition three different glacial periods can be distinguished.

*) Anschrift des Autors: Prof. Dr. G. Wenzens, Geographisches Institut der Universität Düsseldorf, Universitätsstraße 1, 4000 Düsseldorf.
Because of the thickness of the Mosel- and Moselotte-glacier in the Remiremont/St. Amé basin a large diffluence during the Würm is improbable, it may at most have reached to the south of le Tholy area. The maximum heights of the Würmian deposits east of le Tholy prove this view. They can only be deposited by the Vologne-glacier and its lateral glaciers. For the Würm period the snow line was at about $850 \mathrm{~m}$.

The ridges east of le Tholy are of fluvioglacial or lacustrin origin. The Vologne-glacier dammed the melted ice from the Cellet valley, initiating the deposition of thick deltaic sediments. During a phase of melting a delta was formed between the Bas Beillard end moraine and the Vologne-glacier's front. These deltaic sediments of le Costet were subsequently covered by proglacial sediments caused by the Pré Chaussote end moraine.

\section{[La morphologie pleistocène dans la vallée de la Cleurie (Vosges)]}

Résumé: Les recherches sur les dépôts et formes glaciaires et fluvioglaciaires dans la vallée de la Cleurie conduisent aux thèses suivantes concernant l'histoire glaciaire de cette région:

A l'aide des critères: dégradation du modelé glaciaire, degré d'altération et d'encroûtement, position altimétrique et composition des matériaux il est possible de distinguer des dépôts de trois périodes glaciaires.

A cause de l'épaisseur des glaciers de la Moselle et de la Moselotte dans le bassin de Remiremont/St. Amé une langue diffluente peut seulement s'être étendu jusqu'au sud du Tholy. Cela est prouvé par l'altitude des dépôts würmiens à l'est du Tholy qui ont été mis en place par le glacier de la Vologne ou ses petits glaciers tributaires. La limite des neiges würmiennes était à $850 \mathrm{~m}$.

Les rides à l'est du Tholy sont d'origine fluvioglaciaire ou lacustre. Les eaux de fonte de la vallée du Cellet ont été barrées par le glacier de la Vologne où elles ont sédimenté un delta de grande épaisseur. Pendant une phase de fonte un autre delta - celui du Costet - s'est établi entre la moraine terminale de Bas Beillard et le glacier de la Vologne. 
Ses sédiments glacio-lacustres ont été superposés par des dépôts proglaciaires issus de la moraine terminale de Pré Chaussote.

\section{Das Untersuchungsgebiet}

Das Cleurie-Tal entstand als hydrographische Einheit erst während der Würmeiszeit und bildete bis dahin den Unterlauf der Vologne. Diese entspringt an der Westflanke des über $1.300 \mathrm{~m}$ hohen Vogesenkammes, durchfließt die Seen von Retournemer und Longemer sowie die steile Schlucht von Kichompré, biegt südlich Bruyères aus der SE-Richtung kommend fast im rechten Winkel nach SW ab und erreicht in $350 \mathrm{~m}$ Höhe bei Pouxeux die Mosel. Noch zu Beginn des Würms benutzte die Vologne den wesentlich kürzeren Weg über Gérardmer und le Tholy. Während der letzten Vereisung schuf der Vologne-Gletscher das breite Zungenbecken, das heute der See von Gérardmer einnimmt, und hinterließ die im Westen den See aufstauende, aus zwei Wällen bestehende Endmoräne Pré Chaussote (Abb. 1).

Beim Abtauen der Gletscherzunge durchbrachen Schmelzwässer die niedrige Wasserscheide von Kichompré. Durch Akkumulation der abschmelzenden Moräne erhöhte sich der Talboden östlich von Gérardmer; in das nunmehr rückläufige Gefälle schnitt sich die Jamagne als neuer Nebenfluß der Vologne ein, seit

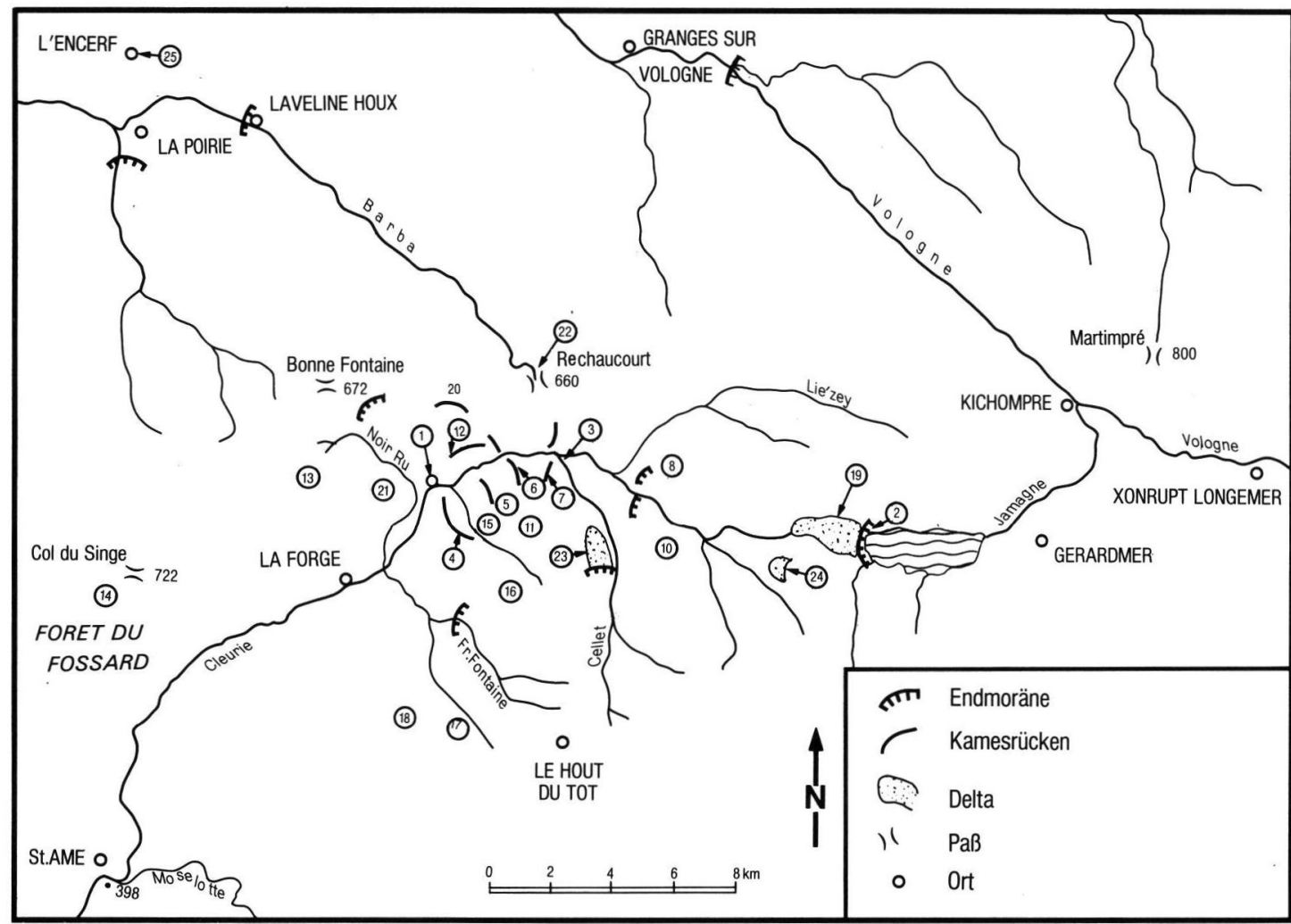

1 Le Tholy

2 Pré Chaussote

3 Rain Brice

4 Noirmont

5 La Goutte Villemin

$6 \mathrm{La}$ Basse

7 Le Bannerot

8 Bas Beillard

9 La Petite Neuvelotte
10 Le Haut Poirot

11 Roche de Lait

12 Le Petit Paradis

13 Faing la Biche

14 Le Hêtre de la Vierge

15 Ferme Berlingoutte

16 Les Quatre-Vents

17 La Croix des Hêtres

18 La Haut des Charmes
19 Le Costet

20 Le Vieux Tholy

21 Blongoutte

22 Trou d'Enfer

23 Pré J'Espère

24 Les Granges Bas

25 St.-Jean-du-Marché

Abb. 1: Übersichtskarte.

Fig. 1: Schéma général. 
dieser Zeit trennt die Endmoräne von Pré Chaussote das Einzugsgebiet der Vologne von dem der Cleurie.

Das ca. $15 \mathrm{~km}$ lange Cleurie-Tal zeichnet sich durch den Wechsel von Talweitungen und -verengungen aus, wobei letztere von mehreren Rücken gequert werden. In dem nur $2 \mathrm{~km}$ langen Engtal zwischen le Tholy und Rain Brice hat sich die Cleurie teilweise um $100 \mathrm{~m}$ in die Wälle von Noirmont (624 m), la Goutte Villemin $(646 \mathrm{~m})$, la Basse $(622 \mathrm{~m})$ und le Bannerot $(612 \mathrm{~m})$ eingeschnitten. Östlich schließt sich ein fast $1 \mathrm{~km}$ breites Becken an, in das auch die beiden wichtigsten Nebenflüsse, Cellet und Liézey, münden. Es wird im E durch den breiten, $660 \mathrm{~m}$ hohen Rücken von Bas Beillard abgeschlossen.

Auch südlich le Tholy weitet sich wieder der Talboden, größere Wälle sind hier nicht mehr ausgebildet. Die Cleurie mündet in knapp $400 \mathrm{~m}$ Höhe in die Moselotte, die nach $4 \mathrm{~km}$ bei Remiremont in die Mosel fließt.

\section{Stand der Forschung}

1966 legte SERET eine umfangreiche Arbeit über die Vergletscherung der Südwestvogesen vor. Er unterscheidet drei verschiedene Vergletscherungsperioden, die er der Mindel-, Riß- und Würmeiszeit zuordnet. Während die älteste Vereisung als Kappenvereisung gedeutet wird, sollen sich Riß- und Würmvergletscherung auf die großen Talzüge beschränkt haben, wobei das Rißeis nur im S weit über die würmeiszeitliche Talvergletscherung hinaus reichte (s. Abb. 2). Die zum Teil über 800 bzw. $1.000 \mathrm{~m}$ aufragenden Wasserscheidenbereiche wären demnach eisfrei gewesen. Der glaziale Formenschatz des Cleurie-Tales wird völlig neu interpretiert: Die Eismassen im unteren CleurieTal sollen vom Moselotte-Gletscher stammen. SERET (1966: 462) nimmt an, daß dieser im Mündungsbereich zur Mosel aufgestaut wurde, so daß sich seine Eismassen durch das Cleurie-Tal bis östlich von le Tholy talaufwärts bewegten, während gleichzeitig der Vologne-Gletscher westlich von Gérardmer stirnte. Eine ähnliche vis-à-vis-Lage beider Gletscherenden soll sowohl in der Riß- als auch in der Würmeiszeit ausgebildet gewesen sein. Die Detailkarte (SERET 1966: Fig. 66) eröffnet weitere Einblicke. Den glazialen Formenschatz des Tales beherrschen die Endmoränen: 6 stammen aus der Würm-, 4 aus der Rißeiszeit. In der Rißkaltzeit näherten sich die Gletscher aus dem Vologne-bzw. Moselotte-Tal bis auf $1.500 \mathrm{~m}$, in der Würmkaltzeit bis auf $5 \mathrm{~km}$. Die Endmoräne Bas Beillard bildet nach dieser Auffassung den äußeren Rand des rißeiszeitlichen, die Endmoräne Pré Chaussote den des würmeiszeitlichen Vologne-Gletschers. Die Herkunft des Eises östlich von le Tholy aus dem
Moselotte-Tal begründet SERET (1966: 475) anhand der nach $\mathrm{E}$ geschwungenen Wallformen und lithologischer Merkmale.

Diesen Auffassungen widerspricht SALOME (1968: 48) in seiner Dissertation über das Moselotte-Gletschersystem. Er lehnt die Existenz einer weitreichenden Moselotte-Diffluenzzunge im Cleurie-Tal ab, da hierfür die Mächtigkeit des Eises im Moselotte-Tal nicht ausgereicht hat. SALOME bestreitet außerdem die Deutung der Wälle bei le Tholy als Endmoränen. Er mißt den fluvioglazialen Prozessen eine wesentlich größere Bedeutung bei und erklärt alle Wälle westlich der Endmoräne Pré Chaussote als Kamesrücken bzw. -terrassen, die ausschließlich von einem würmeiszeitlichen Vologne-Gletscher stammen. Im Gegensatz zu SERET findet SALOME (1968: 25) im Cleurie-Tal keine Hinweise auf eine rißeiszeitliche Talvergletscherung.

In jüngeren Arbeiten geht SERET $(1980,1985)$ leider nicht auf die Einwände SALOMEs ein, sondern modifiziert lediglich das Alter der Vergletscherungen. In Anlehnung an die Pollenstratigraphie des GrandePile Moors hält SERET (1985: 19) nun eine Gleichsetzung der älteren Vereisung mit der Saaleeiszeit, der mittleren mit der älteren Weichseleiszeit und der jüngeren Vereisung mit der jüngeren Weichseleiszeit für wahrscheinlich.

Anfang der 70er Jahre haben FlAGEOLLET \& HAMEURT (1971) die Ablagerungen des Cleurie-Tales erneut untersucht und sind zu folgenden Ergebnissen gelangt: Sowohl an den Flanken als auch am Boden des Cleurie-Tales sind nur wenige Zeugen eines vermutlich älter als würmzeitlichen Glazials erhalten. Nahezu der gesamte Formenschatz stammt aus der Würmeiszeit; bei den Ablagerungen handelt es sich nach den sehr sorgfältigen Untersuchungen von FLAGEOLLET \& HAMEURT vorwiegend um lakustrische Sedimente; für Endmoränen halten sie lediglich den Wall Pré Chaussote und Ablagerungen bei Noir-Ru (1 km nordwestlich von le Tholy). Die Autoren bestätigen die These SERETs, daß sich ein Arm des MoselotteGletschers im Würm cleurieaufwärts erstreckte, nehmen aber an, daß die Zunge noch weiter nach $E$ reichte und bei Bas Beillard auf den Vologne-Gletscher traf. Im Bereich des Zusammenflusses der beiden Gletscherzungen überschritt das $100 \mathrm{~m}$ mächtige Eis die Höhe von $680 \mathrm{~m}$. Der heutige Formenschatz des Tales wurde durch die Ausbildung mehrerer Seen geprägt, die während des Abschmelzens der Eismassen entstanden. Aus der Höhenkonstanz der Ablagerungen schließen FLAGEOLLET \& HAMEURT auf länger andauernde Seespiegelstände im 675-665 m-, 630-625 m-, $600 \mathrm{~m}$ - und $550 \mathrm{~m}-\mathrm{Niveau}$. Die Stufung der Sedimente ist also nicht die Folge von Rückzugsstadien, sondern durch phasenhaftes Absinken der Seespiegelhöhen bedingt. 


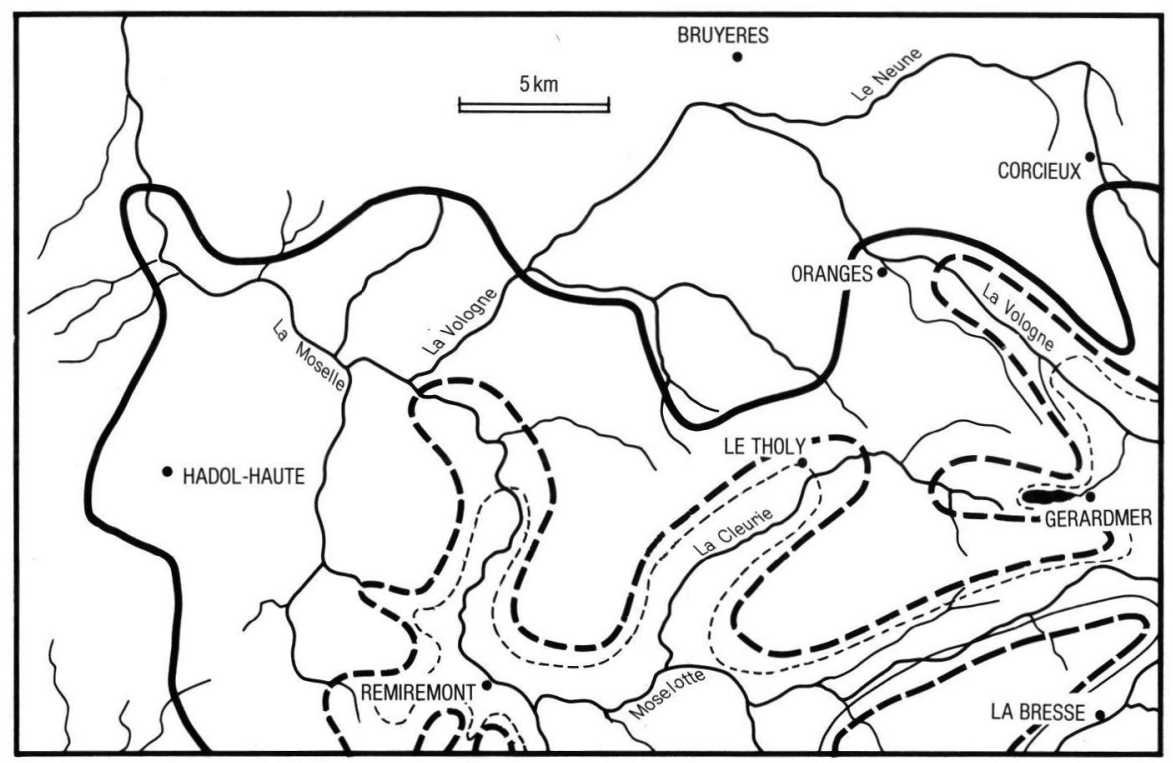

Abb. 2: Grenzen der Vereisungen und stratigraphische Zuordnung.

Fig. 2: Limites des glaciations et corrélation stratigraphique.

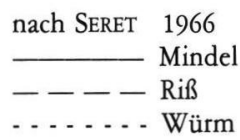

$\begin{array}{ll}1980 & 1985 \\ \text { Ältere } & \text { Saale } \\ \text { Mittlere } & \text { ältere Weichsel } \\ \text { Jüngere } & \text { jüngere Weichsel }\end{array}$

nach SERET (1966: Fig. 72; 1980: Fig. 1; 1985: 19)

\section{Problemstellung}

Auf der Grundlage einer detaillierten Kartierung soll versucht werden, die Vergletscherungsgeschichte des Cleurie-Tales zu rekonstruieren, wobei es zu klären gilt, welches Ausmaß eine eventuelle Moselotte-Diffluenz einerseits und der Vologne-Gletscher andererseits hatten und auf welche Weise beide morphologisch wirksam werden konnten. Bei den Geländearbeiten wurde daher besonderer Wert auf die Ermittlung der höchstgelegenen Zeugen der jüngsten Vergletscherung gelegt. Das Problem, ob der glaziale und fluvioglaziale Formenschatz im Einzugsgebiet des Cleurie-Tales mehreren Kaltzeiten zugeordnet werden kann, wurde deshalb mit Aufmerksamkeit verfolgt. Eine Beantwortung dieser Fragen setzt nicht nur voraus, daß Kriterien zur zeitlichen Differenzierung der Formen und Ablagerungen erstellt werden können, sondern daß auch Aussagen über den jeweiligen Vergletscherungstyp möglich sind.

In den Vogesen wird die Ermittlung der Schneegrenzhöhen durch die Vielzahl von Transfluenzen erschwert. Dies hat dazu geführt, daß vielfach Überlegungen zum Ausmaß der vergletscherten Areale bei der Rekonstruktion der Reliefentwicklung vernachlässigt wurden und der Einfluß lokaler Gletscher unberücksichtigt blieb.

Schließlich wird zu prüfen sein, ob im Untersuchungsgebiet Hinweise auf mehrere Vereisungen vorliegen. Zur Klärung der Frage nach älteren Kaltzeiten wurde die Kartierung nach $\mathrm{N}$ hin ausgedehnt.

$\mathrm{Da}$ die Darstellung einer Reliefentwicklung zunächst jedoch eine den Geländegegebenheiten entsprechende Deutung der glazialen und fluvioglazialen Ablagerungen notwendig macht, wurde der Formenschatz des Cleurie-Tales einer genauen Analyse unterzogen.

\section{Der glaziale und fluvioglaziale Formenschatz des Cleurie-Tales}

Die unterschiedliche Ansprache und Datierung der im Cleurie-Tal ausgebildeten Formen soll an einigen Beispielen diskutiert werden. Eine Schlüsselstellung in bezug auf Genese und Alter nimmt der Wall von B a s B e ill a r d ein. SERET (1966: 466, 472) deutet ihn als Stauchendmoräne, die den weitesten Vorstoß des Vologne-Gletschers während der Rißver- 
eisung markiert und führt als Beleg für das höhere Alter die im Vergleich zu den jüngeren Endmoränen stärkere Überprägung der Hänge an.

Für Flageollet \& HAMEURT (1971: 155) handelt es sich um Ablagerungen mit vorwiegend "moränischem" Charakter, die bei der Berührung von Vologne-Gletscher und Moselotte-Diffluenzzunge während des Würms entstanden sind. Während das Sediment im wesentlichen glazigenen Ursprungs ist, soll die Form durch die während der Abschmelzperiode ausgebildeten Seen geprägt worden sein. Sie ist ein Teil ehemals ausgedehnter Seeterrassen im $675 \mathrm{~m}$ bzw. 630 m-Niveau.

Von dem insgesamt $65 \mathrm{~m}$ hohen Wall sind lediglich die oberen $16 \mathrm{~m}$ aufgeschlossen: An der Basis in 644 $\mathrm{m}$ befinden sich ungestörte Lockersedimente aus zum Teil geschichteten Sanden und ungeregelten Schotterlagen. Sie werden von einer maximal $3 \mathrm{~m}$ mächtigen Moräne ohne Störungen gekappt, deren vorwiegend kantige Blöcke in einer rötlichen, tonigen Matrix fest verbacken sind. Es folgt eine Wechsellagerung aus geschichteten Sanden und Kiesen mit eingeschalteten Schotterlagen, die insgesamt deutliche Stauchungsstrukturen aufweist. Darüber liegt eine $5 \mathrm{~m}$ mächtige Moräne, in der einzelne, aber bis zu $2 \mathrm{~m}$ große Blöcke enthalten sind. Die Matrix dieser Moräne besteht aus unverfestigten, hellen Sanden und Kiesen. Rötlich verwitterte Sande, auf denen eine $40 \mathrm{~cm}$ mächtige Braunerde entwickelt ist, schließen das Profil ab. Der Wall grenzt im S in $675 \mathrm{~m}$ Höhe an den Granitsporn le Haut Poirot, der in 690-695 m eine ca. $200 \mathrm{~m}$ lange und $100 \mathrm{~m}$ breite Verebnung aufweist. Sie ist mit einer dichten Streu von Moränenblöcken überzogen.

Der Rücken von B a s B e ill a r d ist aufgrund der Zusammensetzung des hangenden Materials als $\mathrm{S} \mathrm{t}$ a $\mathrm{u} \mathrm{ch}$ e $\mathrm{nd} \mathrm{m}$ or ä $\mathrm{n}$ e eines erneut vorstoßenden Vologne-Gletschers zu deuten, dessen proglaziale Sedimente gestaucht und anschließend mit einer Satzendmoräne bedeckt wurden.

Auch die Form ist eindeutig als glazigen zu bezeichnen. Die Asymmetrie des Walles sowie die Stauchungsstrukturen weisen auf den Moränencharakter hin. Verebnungen in 620-625 m, wie sie FLAGEOLLET \& HAMEURT (1971: 156) beschrieben haben, sind jedoch als Talbodenreste der Cleurie zu deuten.

Welche Hinweise ermöglichen nun eine Aussage über das A l t e $r$ dieser Moräne? Relative Alterseinstufungen von glazialen und fluvioglazialen Ablagerungen basieren im wesentlichen auf geomorphologischen und pedologischen Kriterien. Daß die Ansprache der Verwitterungsintensität der Sedimente und Böden sehr subjektiv sein kann, wird durch die unterschiedlichen Altersangaben der verschiedenen Auto- ren deutlich (vgl. FlaGEOLIET \& HAMEURT 1971: 130, 146-150, 165 - 166). Die offensichtlich geringe Aussagekraft der Verwitterungsintensität zeigt die voneinander abweichende Zuordnung zu den einzelnen Kaltzeiten in den Arbeiten SERETs von 1966 und 1985 (Abb. 2).

Solange keine absoluten Datierungen möglich sind, kann im Untersuchungsgebiet vor allem der $\mathrm{E} \mathrm{r}$ $\mathrm{h}$ a $\mathrm{lt}$ u n g s z s t a n d der Formen zur Unterscheidung zwischen würmeiszeitlichen und älteren Ablagerungen herangezogen werden. Dieses Merkmal eignet sich im Falle des Cleurie-Tales - durch die speziellen hydrographischen Umstände bedingt - in besonderem Maße als wesentliches Kriterium der zeitlichen Einordnung: So erfolgt die gesamte Entwässerung einschließlich der abgeschmolzenen Eismassen des Vologne-Einzugsgebietes seit Entstehung der Endmoräne Pré Chaussote nicht mehr durch das CleurieTal. Die Erosion ist seitdem westlich der Endmoräne Pré Chaussote auf ein Minimum reduziert, so daß auch die Ausräumung der würmeiszeitlichen Sedimente und die Zerschneidung der Formen nur geringe Ausmaße angenommen hat.

Der gute Erhaltungszustand sowie der schmale Taleinschnitt der Cleurie lassen daher für den Endmoränenrücken Bas Beillard nur ein junges Alter zu.

Obwohl die Cleurie aufgrund der Zuflüsse aus dem Cellet- und Liézey-Tal unterhalb von Bas Beillard über eine größere Erosionskraft verfügt, so daß das Tal bei le Tholy wesentlich breiter ist, sprechen die wenig abgeflachten Hänge der dicht beieinander liegenden Wälle von le Tholy ebenfalls für ein würmeiszeitliches Alter. Dies gilt auch für den Rücken bei $\mathrm{l}$ e $\mathrm{B}$ a n n e r o t, den SERET als Endmoräne der MoselotteDiffluenzzunge einer älteren Kaltzeit gedeutet hat. Wegen seiner geringen absoluten Höhe von $612 \mathrm{~m}$ wirkt er zwar insgesamt breiter und abgeflachter, sein $14^{\circ}$ steiler Osthang erhebt sich jedoch mit deutlichem Knick aus dem Becken von Rain Brice. Mehrere Aufschlüsse und Anschnitte zeigen seinen Aufbau: An der Basis in $600 \mathrm{~m}$ befinden sich schwach geschichtete Schotter aus teilweise gut gerundeten Graniten. Es folgt ein $2 \mathrm{~m}$ mächtiges Paket aus feingeschichteten Kiesen, Sanden und einzelnen Schotterlagen, die mit $12^{\circ}$ nach Osten einfallen. Es wird von einem $60 \mathrm{~cm}$ mächtigen Grobschotterkörper überlagert, der seinerseits von einer $1,5 \mathrm{~m}$ mächtigen Solifluktionsdecke gekappt wird.

Der nördliche Flügel zeigt zwischen 590 und 595 m ebenfalls mehrere Meter gut geschichtete, helle Sande, in die einzelne Taschen aus ungeschichteten Kiesen und Geröllen eingelagert sind. Der Wall von le Bannerot weist somit keinerlei Merkmale auf, die auf eine Endmoräne schließen lassen. Zusammensetzung 
und Schichtung des Materials sprechen vielmehr für eine fluvioglaziale Ablagerung zwischen Toteismassen; der nördliche Flügel zeigt lakustrische Sedimentationsbedingungen an.

Bei den übrigen W älle $\mathrm{n}$ von le $\mathrm{Th}$ oly gewähren nur noch kleinräumige Anschnitte Einblick in die Ablagerungen, die jedoch stets aus mehr oder weniger deutlich geschichteten Lockersedimenten bestehen, wobei helle Feinsande im allgemeinen überwiegen. Zwischengeschaltete Schotterlagen bilden die Ausnahme, Moränenblöcke treten nur vereinzelt auf. Lediglich der Wall la Goutte Villemin ist mit auffallend großen Blöcken bedeckt, wobei nur wenig Gesteinsvarietäten vorkommen. $\mathrm{Da}$ oberhalb des Rückens der Steilhang von „Roche de Lait” mit einem Blockstrom aus diesem Material einsetzt, ist eine solifluidale Umlagerung der Blöcke nicht auszuschließen. FLAGEOLLET \& HAMEURT (1971: 158) halten es für möglich, daß die Felsen aus dem Anstehenden stammen.

In Übereinstimmung mit SALOME (1968: 37) und FLAGEOLIET \& HAMEURT (1971: 160) ist die Deutung der Wälle von le Tholy als Endmoränen auszuschlieBen. Hinweise, die ein unterschiedliches Alter der Ablagerungen nahelegen, fehlen ebenfalls.

FlageOlLET \& HAMEURT (1971: 165) haben an verschiedenen Stellen stark verfestigtes Material („Crassin”) beschrieben. Die Autoren schließen nicht aus, daß es sich um Relikte einer älteren, eigenständigen Glazialepoche handelt, halten es jedoch auch für möglich, daß der „Crassin” einer frühwürmzeitlichen Vergletscherungsphase angehört. Die Untersuchungen legen eine Differenzierung der verfestigten Moränen nahe: Im Talbodenbereich liegen sie meist zwischen geschichteten Sanden und Kiesen (z. B. Aufschluß Bas Beillard, in $540 \mathrm{~m}$ an der Basis des Rückens Noirmont, in $590 \mathrm{~m}$ Höhe südlich le Petit Paradis). Für diese Vorkommen halte ich ein frühwürmeiszeitliches Alter für wahrscheinlich und betrachte sie als Grundmoräne der Talvergletscherung. Sie werden in dieser Position stets von fluvioglazialen bzw. limnischen Sedimenten bedeckt, wohingegen verfestigte Moränen im höheren Hangbereich unmittelbar an die Oberfläche treten und von Solifluktionsschutt überzogen sind.

So wird das Seitental Fro ide Fon t a i n e in rund $640 \mathrm{~m}$ Höhe von einem Wall abgeriegelt, an dessen distalem Ende Moräne in grauer, verfestigter Matrix aufgeschlossen ist. Die oberen $50-100 \mathrm{~cm}$ sind extrem stark verbacken und verwittert, das Bindemittel zeigt außerdem eine rötliche Färbung. Das Material ist scharf gegen den hangenden, lockeren Solifluktionsschutt abgegrenzt.
Diese Moräne unterscheidet sich durch den starken Verkittungsgrad der Ablagerungen und die insgesamt sehr verwaschene Form von den würmeiszeitlichen Moränen, so daß ein rißeiszeitliches Alter naheliegt. SERET (1966: 473) hat sie ebenfalls als rißeiszeitlich gedeutet und als Beleg für eine auch im vorletzten Glazial ausgebildete Moselotte-Diffluenz gewertet. Letztere Interpretation läßt sich jedoch mit zwei Gegebenheiten nicht vereinbaren:

1. Die Moräne setzt sich zu $90 \%$ aus Gesteinen zusammen, die im Einzugsgebiet des Froide Fontaine-Baches anstehen.

2. Der Moräne ist talwärts eine flach abfallende Verebnung in $620 \mathrm{~m}$ Höhe vorgelagert. Ein Schurf ergab unter einer Braunerde schwach geschichtete Kiese und Feinsande, die insgesamt zum CleurieTal hin einfallen und die einen mehrfachen Farbwechsel von Grau und Ocker aufweisen. Diese Sedimente bauen somit einen der Endmoräne vorgelagerten Sander auf.

Bei den Ablagerungen in $640-660 \mathrm{~m}$ handelt es sich demnach um die Endmoräne eines Seitengletschers, dessen Einzugsgebiet mit dem des Froide FontaineBaches übereinstimmt.

Moränen, die sich durch einen hohen Verfestigungsgrad auszeichnen und von rötlichen Schlieren durchzogen sind, befinden sich meist oberhalb des eigentlichen Talbereiches: $z$. B. auf der $710 \mathrm{~m}$ hohen Verebnung Faing la Biche (westlich le Tholy), am 720 m hohen Paß le Hêtre de la Vierge (nördlich Commune de Cleurie), in $700 \mathrm{~m}$ an der Straße von Berlingoutte nach les Quatre-Vents. Sie sind einer älteren Vergletscherung zuzuordnen, die sich jedoch sonst nur durch eine mehr oder weniger dichte Blockstreu nachweisen läßt. Größere Aufschlüsse befinden sich u. a. nördlich der Cleurie im $800 \mathrm{~m}$ hohen F or êt D o m a $\mathrm{n}$ i a le de Fossard (nordöstlich von Remiremont), südlich des Höhenpunktes 779,8 am Fardeau St. Christoph, am Forstweg östlich des $808 \mathrm{~m}$ hohen Tête du Houssot sowie südlich des Flusses auf dem bis $\mathrm{zu} 1.000 \mathrm{~m}$ hohen Rücken von $\mathrm{le} \mathrm{H} \mathrm{a} \mathrm{u} \mathrm{t} \mathrm{d} \mathrm{u}$ $\mathrm{T}$ ô $\mathrm{t}, \mathrm{u}$. a. in $800 \mathrm{~m}$ Höhe nördlich la Croix des Hêtres sowie in $810 \mathrm{~m}$ südlich le Haut des Charmes.

\subsection{Die würmeiszeitliche Vergletscherung}

Die Zusammenschau verschiedener Geländebefunde bezüglich der jeweils höchstgelegenen Zeugen würmeiszeitlicher Vergletscherung stellt sich folgendermaßen dar:

Am Waldweg zwischen le Pré Chaussote und Liézey befindet sich nach $500 \mathrm{~m}$ in $700 \mathrm{~m}$ Höhe ein Anschnitt mit junger Moräne. Diese ist auch im Stein- 
bruch nördlich le Costet in gleicher Höhenlage aufgeschlossen. Die dichte Blockstreu auf dem $690 \mathrm{~m}$ hohen Sporn des Haut Poirot kann mit Vorbehalten als gleichaltrig betrachtet werden.

Nördlich von le Vieux Tholy markiert ein Wall in 665 $\mathrm{m}$ ebenso eine Gletscherbegrenzung wie die gegenüberliegenden fluvioglazialen Sedimente in $655 \mathrm{~m}$ oberhalb der Ferme Berlingoutte.

Ein Lobus war in das Tal Noir-Ru vorgestoßen und hinterließ dort eine Endmoräne bei la $\mathrm{Pe}$ t i t e $\mathrm{Ne} u \mathrm{v}$ e lot t e. Die Oberkante der in heller, sandig-kiesiger Matrix befindlichen Blöcke reicht bis in ca. $630 \mathrm{~m}$. Ein sich daran anschließender Sander aus flach nach NW einfallenden geschichteten Kiesen und Sanden weist an der Oberfläche abfluß- lose Hohlformen auf, die als ehemalige Toteislöcher angesprochen werden können. Der $672 \mathrm{~m}$ hohe Paß Bonne Fontaine wurde demnach von den würmeiszeitlichen Eismassen nicht mehr erreicht. Hier findet man lediglich eine lockere Moränenstreu vor.

Östlich des Gehöftes Blongoutte (südwestlich le Tholy) konnte in $625 \mathrm{~m}$ eine geringmächtige Moräne nachgewiesen werden, während die oberhalb gelegene kleine Verebnung in $650 \mathrm{~m}$ nur vereinzelte Blöcke trägt.

Im unteren Talbereich der Cleurie markiert die aus dem Froide Fontaine-Tal beschriebene rißeiszeitliche Endmoräne mit dem vorgelagerten Sander in $620 \mathrm{~m}$ ü. M. eine maximale Höhe, die von der würmeiszeitlichen Talvergletscherung nicht mehr erreicht wurde.

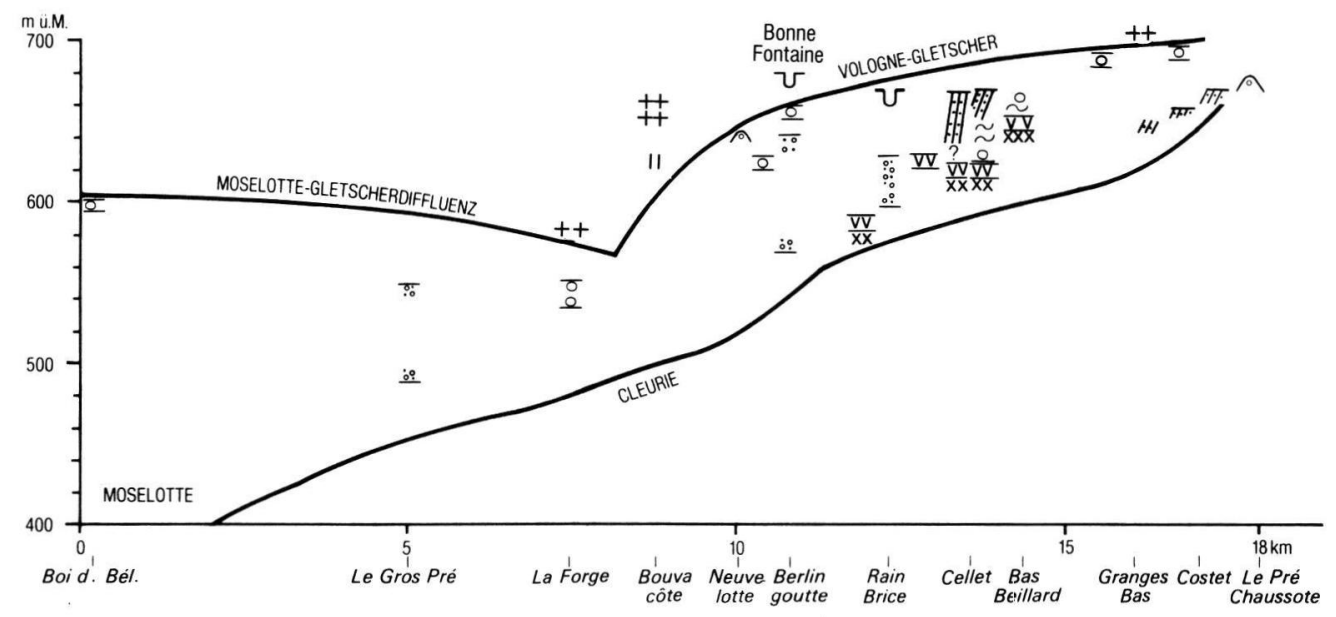

บ Paß Rechaucourt

Riß

$++\quad$ Moräne (verfestigt)

II Fluvioglaziale Sedimente

Würm

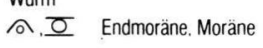

$\because$ Kames

$\check{\simeq}$ gestauchte Kames

$\equiv \quad$ lakustre Sedimente. Sander

$\pi \%$ Deltasedimente mit

$\%$ und ohne Schotterkappe

V V Moräne (Crassin)

XX Fluvioglaziale Sedimente

Abb. 3: Rekonstruktion der würmeiszeitlichen Gletscheroberfläche im Cleurie-Tal unter Berücksichtigung der Ausdehnung einer möglichen Moselotte-Diffluenzzunge.

Fig. 3: Reconstruction de la surface glaciaire würmienne dans la vallée de la Cleurie. 
Der $550 \mathrm{~m}$ hohe Rücken östlich la Forge gibt hier eine obere Grenze der Vergletscherung an.

Die Ergebnisse der Kartierung und die Analyse der Aufschlüsse lassen nun eine Rekonstruktion der würmeiszeitlichen Vergletscherung des Cleurie-Tales $\mathrm{zu}$, wobei alle aufgeführten Geländebefunde im Widerspruch zu einer großräumigen Moselotte-Diffluenzzunge stehen, die sich nach SERET $(1966,1985)$ und FlaGEOLLET \& HAMEURT (1971) bis weit östlich von le Tholy erstreckt haben soll und deren Gletscheroberfläche dort noch bis $680 \mathrm{~m}$ ü. M. gereicht haben müßte (s. u.).

Die Höhenlage der jeweils höchsten würmzeitlichen Vorkommen nimmt von der Endmoräne Pré Chaussote nach la Forge cleurieabwärts deutlich und kontinuierlich von $700 \mathrm{~m}$ auf $550 \mathrm{~m}$ ü. M. ab (s. Abb. 3), was auf eine in E-W-Richtung vorstoßende Gletscherzunge hinweist. Unabhängig von den eigenen Beobachtungen und Schlußfolgerungen sprechen auch folgende Überlegungen gegen eine weit ins Cleurie-Tal hineinreichende Diffluenzzunge (Abb. 4):

Berücksichtigt man nämlich die Entfernung von über $10 \mathrm{~km}$ zwischen Moselotte-Tal und dem Ende der vermeintlichen Diffluenzzunge, so hätte die Oberkante des Mosel-Gletschers und damit auch des MoselotteGletschers im Becken von Remiremont mindestens $750 \mathrm{~m}$ ü. M. gelegen haben müssen, damit ein minimales Oberflächengefälle für den Eistransport cleurieaufwärts überhaupt entstehen konnte. In diesem Fall wäre der Mosel-Gletscher bei Remiremont $(390$ m ü. M.) über $350 \mathrm{~m}$ mächtig gewesen. Die Eismassen bei Remiremont können aber nur weniger als $200 \mathrm{~m}$ mächtig gewesen sein, da die Endmoräne la Demoiselle-Hautmantarde $4 \mathrm{~km}$ westlich von Remiremont in maximal $560 \mathrm{~m}$ Höhe, also ca. $170 \mathrm{~m}$ über dem Talboden liegt. Eine Mächtigkeit des Mosel-Gletschers von ca. $180 \mathrm{~m}$ in diesem Raum ergibt sich aus den Überlegungen von SERET (1966: 275, Fig. 22), der aus der Höhe vergletscherter und unvergletscherter Pässe im Bereich des kleinen Vogesenkammes die Eisdicke ermittelte und die Oberkante bei Remiremont in ca. $580 \mathrm{~m}$ Höhe ansetzt. Der weiteste würmeiszeitliche Vorstoß des Mosel-Gletschers bei Noirgeux befindet sich auch in nur $6 \mathrm{~km}$ Entfernung vom Diffluenzbereich.

Geht man einmal von der - allerdings wenig wahrscheinlichen - Annahme aus, daß der MoselotteGletscher an seiner Mündung in die Mosel gestaut wurde und sich so die Eismassen einen Weg cleurieaufwärts suchen mußten, können sie selbst bei einer nur geringen Neigung der Gletscheroberfläche allenfalls bis auf die Höhe von la Forge gereicht haben. Der dort ausgebildete, nach $\mathrm{N}$ gewölbte $550 \mathrm{~m}$ hohe
Rücken könnte der Form nach als Endmoräne einer Diffluenzzunge gedeutet werden; nach SALOME (1968: 50) handelt es sich jedoch um eine Kamesterrasse.

Eine bis östlich von le Tholy vorstoßende MoselotteDiffluenz ist somit auch aufgrund der Eismächtigkeiten des Mosel- und Moselotte-Gletschers auszuschließen.

Die morphologischen Gegebenheiten im Cleurie-Tal lassen sich ebensowenig mit der Existenz einer Diffluenzzunge vereinbaren. Im Gegensatz zu den Verhältnissen westlich von Remiremont, wo mehrere Endmoränenbögen und zwischengeschaltete lakustrische Sedimente das Oszillieren einer Gletscherzunge belegen, fehlen im Cleurie-Tal entsprechende Zeugen eines mehrfachen Vorstoßes. Die Wälle östlich von le Tholy sind - wie es SALOME (1968: 37) und FlAGEOLLET \& HAMEURT (1971: 145) beschrieben haben - eindeutig fluvioglazialer bzw. lakustrischer Entstehung, so daß auch ihre teilweise nach E gewölbte Form nicht als Beleg für einen Gletschervorstoß in östlicher Richtung angeführt werden kann (vgl. Flageollet \& HameurT 1971: 146).

Gegen ihre Deutung als Endmoräne einer MoselotteDiffluenzzunge spricht noch ein weiterer morphologischer Gesichtspunkt: Hätte ein Gletscher während einer längeren Zeit das Cleurie-Tal abgedämmt, so hätten sich an seiner Stirn die aus dem Liézey-, Celletund Vologne-Gletscher abfließenden Schmelzwasser gestaut und ihre mitgeführten Sedimente abgelagert. Solche Sedimente sind aber im Cleurie-Tal nirgendwo erhalten, und ihre spätere Ausräumung kann, wie bereits dargelegt, ausgeschlossen werden. Außerdem lassen die steilen Fronten der Wälle sowie das breite und tiefe Becken zwischen Rain Brice und der Endmoräne Bas Beillard einen solchen Stau ausschließen.

Als weiteren Beleg für eine Diffluenzzunge haben SERET (1966: 464) und FlageOLLET \& HAMEURT (1971: 137) die petrographischen Beson $\mathrm{d}$ e $\mathrm{r}$ h e it e $\mathrm{n}$ der Ablagerungen angeführt. Sie beziehen sich u. a. auf das Fehlen von Rhyolithgeschieben in den Sedimenten westlich von Bas Beillard. Der $\mathrm{Rh}$ y o l i t h a n t e il in der Endmoräne Pré Chaussote beläuft sich auf $7 \%$, in der Moräne Bas Beillard auf $4 \%$, im Rücken le Bannerot auf $2 \%$ (FlAGEOlLET \& HAMEURT 1971: 135). Es ist deshalb durchaus einleuchtend, wenn Rhyolithgeschiebe weiter talabwärts nicht mehr nachweisbar ist. So haben FLAGEOLLET \& HAMEURT (1971: 135) bei Geschiebezählungen im Bereich des Endmoränenkomplexes des Vologne-Gletschers südlich Granges sur Vologne dreimal kein und zweimal zwei Prozent Rhyolithanteil festgestellt. 


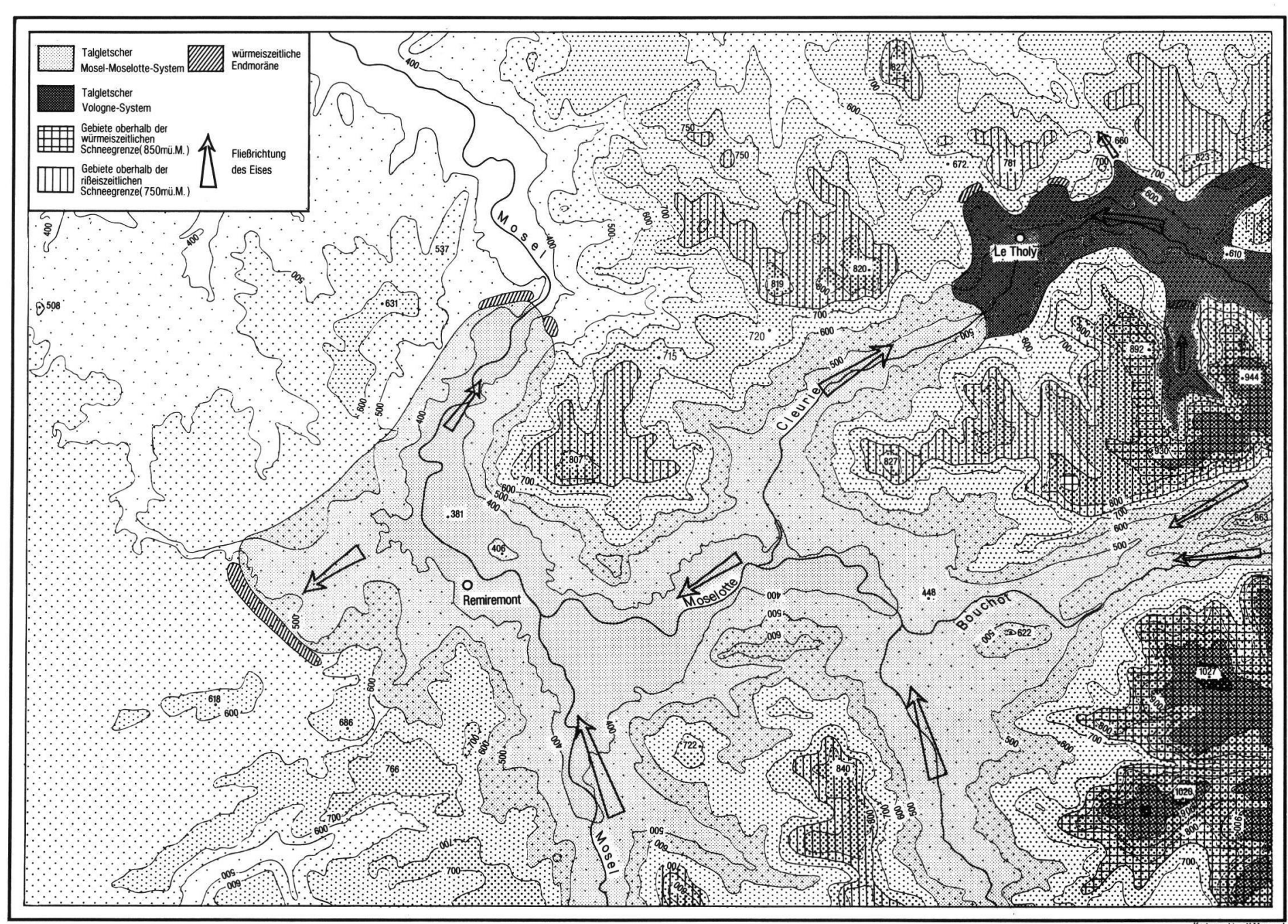

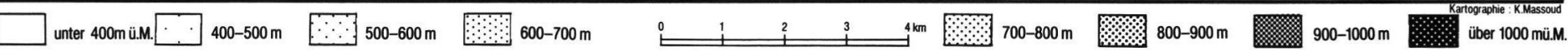

Abb. 4: Höhenschichten, Gletschersysteme und Schneegrenzen im Vologne-, Moselotte- und Moselbereich.

Fig. 4: Niveaux d'altitude, systèmes glaciaires et limites de neige dans les régions de la Vologne, Moselotte et Moselle. 
Das Fehlen von Rhyolith-Geröllen im unteren Talabschnitt der Cleurie spricht übrigens eher gegen eine Moselotte-Diffluenz als dafür. Vergleicht man nämlich auf der Geologischen Karte $1: 100.000$ (HAMEURT 1967) das nur sporadische Vorkommen im oberen Vologne-Tal mit dem wesentlich größeren Auftreten von Rhyolith im Einzugsgebiet der Moselotte - insbesondere an der Mündung des Chajoux-Tales in die Moselotte -, so wäre nämlich eine Zunahme dieser Gesteinskomponente in den Ablagerungen einer Moselotte-Diffluenzzunge zu erwarten. Die petrographische Zusammensetzung der Moränen in den großen Tälern sagt jedoch über die Herkunft der einzelnen Bestandteile wegen der zahlreichen Transfluenzen und der Aufarbeitung von Ablagerungen einer älteren Kappenvereisung nur wenig aus. Hinzu kommt, daß es sich im Cleurie-Tal im wesentlichen um fluvioglaziale bzw. lakustrische Sedimente handelt, die meist aus der unmittelbaren Umgebung stammen FLAGEOLLET \& HAMEURT 1971: 138).

Da weder die morphologischen Gegebenheiten noch die theoretischen Überlegungen für eine bis östlich von le Tholy vorstoßende Moselotte-Diffluenzzunge sprechen, soll im folgenden versucht werden, die verschiedenen Formen und Ablagerungen neu zu interpretieren. Südlich le Tholy sind keine Ablagerungen erhalten, die als Endmoräne den maximalen Vorstoß des Vologne-Gletschers belegen, deshalb muß davon ausgegangen werden, daß Moselotte- und VologneGletscher ineinander übergegangen sind, ohne markante morphologische Spuren zu hinterlassen. Es ist jedoch zunächst noch zu überprüfen, ob der VologneGletscher überhaupt ein entsprechend großes und hochgelegenes Einzugsgebiet hatte, um so weit vorzustoßen.

Aus der Position der Endmoräne am Ausgang des Cellet-Tales läßt sich je nach der für die Berechnung zugrunde gelegten Methode eine Firnlinie zwischen 780 und $820 \mathrm{~m}$ ermitteln. Da es sich jedoch um ein relativ geschütztes, nordexponiertes Einzugsgebiet handelt, habe ich für den Untersuchungsraum einen Schneegrenzwert von $850 \mathrm{~m}$ zugrunde gelegt. Er entspricht den Angaben von HANTKE (1978: 402), der diese Höhe für den inneren Stand ansetzt.

Geländebefunde belegen den ursprünglichen Kontakt zwischen den Eismassen des Vologne- und CelletGletschers: Die verfestigte Moräne setzt sich unter den Deltaablagerungen bis ins Cleurie-Tal fort. Während der maximalen Würmvereisung kann man also von einer etwas tiefer gelegenen Schneegrenze ausgehen.

Da der Vologne-Gletscher außerdem vom LiézeyGletscher gespeist wurde, hat zu dieser Zeit ein ausreichend großes Nährgebiet existiert, um die Eis- massen im Cleurie-Tal zwischen Bas Beillard und le Tholy bis zu der aus glazialen und fluvioglazialen Ablagerungen rekonstruierten Gletscheroberfläche ansteigen zu lassen.

Nach dem Maximum der Vergletscherung schmolz die Front des Cellet-Gletschers in den Talmündungsbereich zurück, während das Cleurie-Tal selbst noch von Eismassen verfüllt war. Dadurch konnten die Schmelzwässer aus dem Cellet-Tal nicht abfließen; zwischen dem Vologne-Gletscher und der CelletGletscherzunge bildete sich ein Eisstausee. Die Sedimentationsprozesse während dieses Stadiums zeigt Abb. 5: Über einer verfestigten Moräne befinden sich horizontal liegende Warventone, darauf steil einfallende, sandig-kiesige Schichten im Wechsel mit Schotterlagen sowie ein diese Sedimente kappender, schwach talwärts einfallender, $2 \mathrm{~m}$ mächtiger Schotterkörper. Zusammensetzung und Einfallen dieser Schichten, die über einen längeren Zeitraum aufgeschlossen waren, entsprechen einem klassischen Delta, dessen Oberkante zur Ablagerungszeit der hangenden Schotterkappe einen Seespiegel von 668-670 m anzeigt. Sie markiert den Zeitpunkt, zu dem sich der See einen Ablauf über die abschmelzenden Eismassen des Vologne-Gletschers zum $P$ a $B$ v o n $R$ e c h a u c o u r t geschaffen hat. Dadurch wurde im Zuge starker Erosion das canyon-ähnliche Tal des Barba mit der $100 \mathrm{~m}$ tiefen Klamm Trou d'Enfer gebildet und der $\mathrm{Pa}$ auf seine heutige Höhe von $660 \mathrm{~m}$ ü. M. erniedrigt. Die Ursache für dieses Überlaufen des Sees kann in einem erneuten Vorstoß des Cellet-Gletschers zu sehen sein, da die Deltaschichten deutliche Stauchungsstrukturen aufweisen. Während der Entwässerung des Sees über den $\mathrm{Paß}$ von Rechaucourt kam es im Deltabereich zur Ablagerung der hangenden Schotterkappe, deren diskordante Lage und Zusammensetzung aus wesentlich gröberem Material sie nicht als top set beds, sondern als proglaziale Sedimente aufweist. Sie zeigen eine Aktivierung des Abflußgeschehens an. In etwa gleichzeitig mit der Aufschüttung des $\mathrm{Delt}$ a s be i $\mathrm{P} r$ é J'Es p è $\mathrm{r}$ e sind zwischen le Tholy und le Rain Brice die $\mathrm{K}$ a $\mathrm{m}$ e s $\mathrm{r}$ ü c k e $\mathrm{n}$ entstanden. Es handelt sich um Verfüllungen von Zwischenräumen der in Toteisblöcke aufgelösten Zunge des Vologne-Gletschers. SALOME (1968: 50) und FLAGEOLLET \& HAMEURT (1971: 158-160) haben ausführlich den Zusammenhang zwischen geringer Eismächtigkeit auf Spornen und der dadurch begünstigten Ablagerung von fluvioglazialen und lakustrischen Sedimenten in den zuerst eisfreien Talengen dargelegt.

Das Absinken der Schneegrenze auf die ermittelte Höhe von ca. $850 \mathrm{~m}$, das zum erneuten Vorstoß des Cellet-Gletschers führte, hat sich - mit einer gewissen Zeitverzögerung - auch im Cleurie-Tal ausge- 


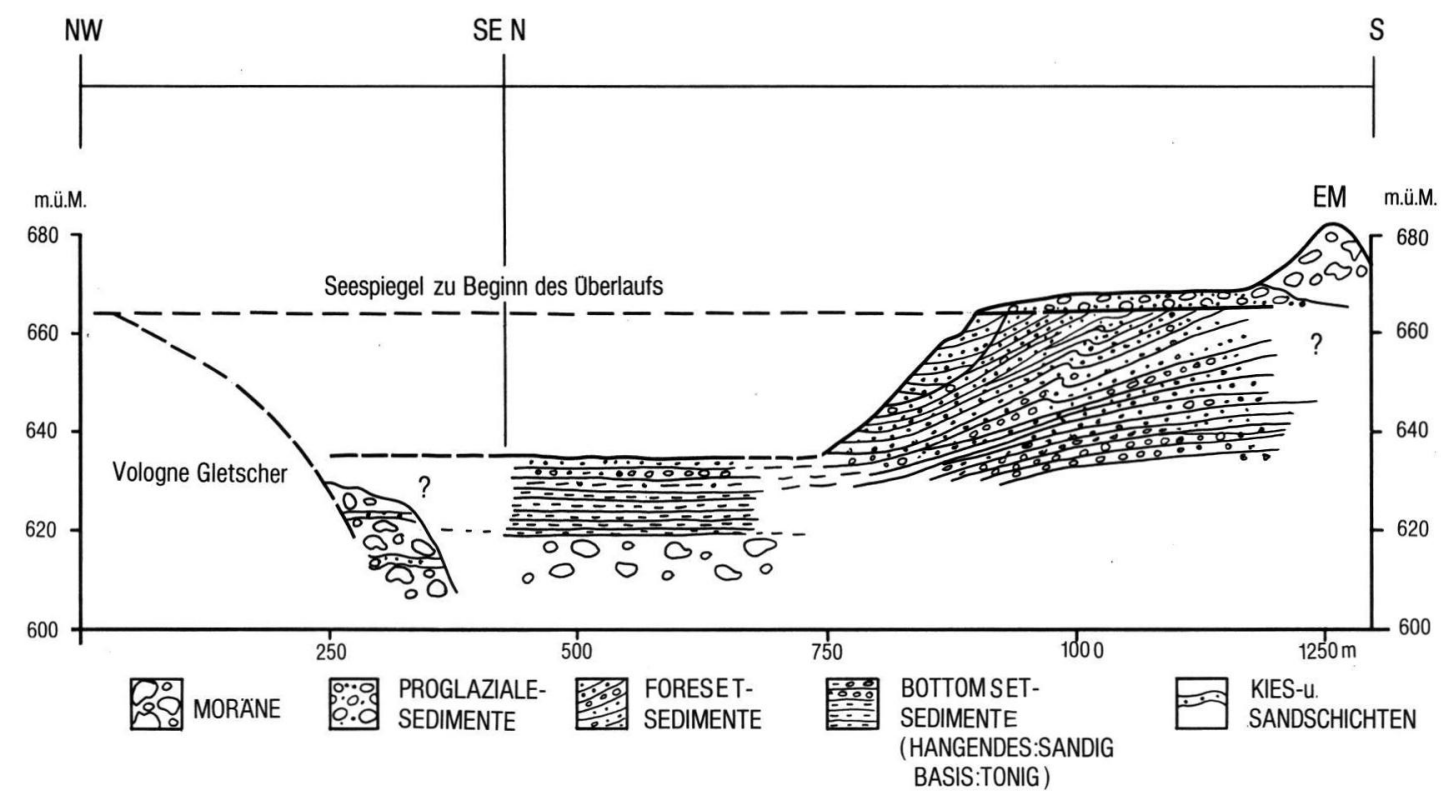

Abb. 5: Aufschlußserie nördlich Pré J’Espère.

Fig. 5: Série de coupes au nord du Pré J'Espère.

wirkt. In dieser Phase wurde die $\mathrm{E} n \mathrm{~d} m$ or ä $\mathrm{n}$ e $\mathrm{B}$ a s $\mathrm{B}$ e i 11 a r d gebildet.

Während der folgenden Abschmelzperiode kam es zur Ablagerung der Deltasedimente von le Costet. Die fore set beds, die aus bis zu $25^{\circ}$ nach W einfallenden Sand-, Kies- und Schotterlagen bestehen, werden von einem schwach geschichteten, ca. $2 \mathrm{~m}$ mächtigen Schotterpaket überlagert, das an der Endmoräne Pré Chaussote in $670 \mathrm{~m}$ ansetzt und im distalen Bereich bis auf $660 \mathrm{~m}$ abfällt. In einem Aufschluß $50 \mathrm{~m}$ vom $688 \mathrm{~m}$ hohen Endmoränenrücken entfernt befinden sich auf horizontalen top set beds $\mathrm{ca} .40 \mathrm{~cm}$ mächtige Sandschichten, die von groben Blöcken überlagert werden. Das völlig ungestörte Erscheinungsbild der top set beds legt nahe, daß das Moränenmaterial nur aufgerutscht ist.

Die Ablagerung der Deltasedimente von le Costet setzt somit einen See voraus, dessen Spiegelhöhe ca. $660 \mathrm{~m}$ betrug.

Die Endmoräne bei Bas Beillard hatte damals die Schmelzwässer aufgestaut, bis sie schließlich durchbrochen wurde. Noch heute befinden sich große Teile über $650 \mathrm{~m}$, in den Randbereichen sogar in $660 \mathrm{~m}$.

Bezüglich der Ursache des Staueffektes, der zur Ausbildung des Deltas von le Costet geführt hat, liegen stark voneinander abweichende Auffassungen vor: Während HoL (1940) ebenfalls die Endmoräne Bas Beillard für die Barriere hielt, nahm
SALOME (1968: 45-49) an, daß Toteisblöcke den Stau verursachten; SERET (1985: 39) wies der MoselotteDiffluenzzunge diese Rolle zu. Sie soll während der jüngsten Vereisungsphase einen $5,5 \mathrm{~km}$ langen See von la Basse bis zum Vologne-Gletscher bei Pré Chaussote aufgestaut haben. Während der Maximalausdehnung soll der See über den Col de Rechaucourt abgeflossen sein, dessen Höhe SERET ebenso wie das Delta von Costet mit $664 \mathrm{~m}$ ü. M. angibt. In der vorhergehenden "mittleren Vereisung" soll unter ganz ähnlichen Bedingungen das Delta von Pré J'Espère entstanden sein, wobei der $\mathrm{Paß}$ von Rechaucourt noch eine Höhe von $680 \mathrm{~m}$ hatte. Die Erniedrigung der Paßhöhe um $16 \mathrm{~m}$ auf das Niveau des Deltas von Costet soll während des Gletschervorstoßes in der ,jüngeren Vereisung" eingetreten sein. Die für eine solche Erniedrigung der Paßhöhe erforderliche Zeitspanne betrachtet SERET (1985: 40) als Beleg für eine zweimalige Talvergletscherung.

Bei dieser Deutung bleiben m. E. folgende Fragen ungeklärt:

1. Durch welchen Prozeß kann in einem Interstadial eine Paßerniedrigung von $16 \mathrm{~m}$ erfolgen?

2. Warum hat sich während der ,mittleren Vereisung" nur ein Delta in der Mündung des CelletTales, das nach SERET nicht vergletschert war, gebildet und nicht vor dem Vologne-Gletscher, wie dies in der jüngsten Vereisungsphase der Fall war? 
3. Wie ist das große Becken von Rain Brice zwischen den Endmoränen der ,,mittleren Vereisung” zu erklären? Hier waren der Auffassung SERET's zufolge die Schmelzwässer des Vologne-Gletschers gestaut, doch sind offensichtlich keinerlei Ablagerungen hinterlassen worden.

4. Während der jüngsten Seebildung im $664 \mathrm{~m}$ Niveau hätten auch die in diesen See mündenden Bäche Liézey und Cellet entsprechend große Deltas aufbauen müssen. Tatsächlich finden sich aber nur zwischen den Endmoränen Bas Beillard und Pré Chaussote weitere deltaartige Sedimente, so z. B. bei les Granges Bas.

Die Erklärungen von FLAGEOLLET \& HAMEURT (1971: 174-176), die in sich schlüssiger erscheinen, seien nur kurz erwähnt: Während die Moselotte-Diffluenzzunge über einen längeren Zeitraum im Bereich des Rückens Bas Beillard verharrte, soll der VologneGletscher bis zur Höhe der Endmoräne Pré Chaussote abgeschmolzen sein, wobei dort das Delta von le Costet entstand. Der See soll sich am nördlichen Rand der Moselotte-Diffluenz bis zum $\mathrm{Paß}$ von Rechaucourt, der ihm als Überlauf diente und auch seine Spiegelhöhe bestimmte, ausgedehnt haben. Erst anschließend sollen die Ablagerungen bei Pré J'Espère sedimentiert worden sein. Auf die Widersprüche, die sich aus der unterschiedlichen Höhenlage der kappenden Schotterdecke beider Deltaablagerungen ergeben, gehen die Autoren nicht näher ein.

Sowohl die Entstehung des Deltas am Ausgang des Cellet-Tales als auch vor der Endmoräne Pré Chaussote läßt sich m. E. ohne die Existenz einer oder mehrerer großräumiger Moselotte-Diffluenzzungen erklären. Belege für deren nur geringe Ausdehnung im Cleurie-Tal wurden bereits aufgeführt.

\subsection{Zum Problem der älteren Vergletscherungen}

Die Rekonstruktion der würmeiszeitlichen Talvergletscherung zeigte, daß die im Cleurie-Tal oberhalb von $700 \mathrm{~m}$ vorkommenden Moränen und Erratika mindestens einer älteren Vereisung angehören. Solche Ablagerungen treten im gesamten Einzugsgebiet der Cleurie auf (s. Kap. 4). Es soll nun die Möglichkeit überprüft werden, sie mehreren Kaltzeiten zuzuordnen.

Im weiteren Untersuchungsgebiet markiert im Moseltal die Endmoräne bei $\mathrm{P}$ o u x e u x den weitesten rißeiszeitlichen Vorstoß des Mosel-Moselotte-Gletschersystems. Da sie sich nur knapp $6 \mathrm{~km}$ talabwärts der würmeiszeitlichen Endmoräne Noirgeux befindet, liegt der Schluß nahe, daß sich Ausdehnung und Typ beider Vergletscherungen nur geringfügig unterscheiden. Diese Überlegung findet sich durch die Lage der rißeiszeitlichen Endmoräne im Froide Fontaine-Tal bestätigt. Im Bereich dieses Talgletschers wäre die rißeiszeitliche Firnlinie bei ca. $750 \mathrm{~m}$ anzusetzen, einem Wert, der auffallend gut der in der Literatur genannten Höhe von 760-790 m entspricht (HANTKE 1978). Die Tieferlegung der Schneegrenze um etwa $100 \mathrm{~m}$ im Vergleich zum Würm hätte das Nährgebiet des Vologne-Gletschers im Cleurie-Tal so vergrößert (s. Abb. 4), daß die Eismassen die beiden Pässe Rechaucourt und Bonne Fontaine überflossen hätten. Die jenseits der Pässe eingetieften Täler zeigen deutliche Merkmale einer glazialen Überprägung; die zugehörigen Endmoränen befinden sich bei la Poirie und nördlich Laveline-du-Houx in ca. $450-470 \mathrm{~m}$. Einzelne Graniterratika auf der aus Buntsandsteinkonglomerat bestehenden $550 \mathrm{~m}$ hohen Einsattelung des Rückens l'Encerf südlich von St.-Jean-du-Marché müssen daher einer weiteren Vergletscherungsphase zugeordnet werden, deren Endmoränen südlich Docelles unmittelbar vor der Mündung des Barba in die Vologne nachgewiesen wurde.

Auch die Moränen und Erratika auf dem $800 \mathrm{~m}$ hohen Forêt Domaniale östlich Remiremont sowie auf dem $820 \mathrm{~m}$ hohen Forêt de Fossard können nicht aus der rißeiszeitlichen Vergletscherung stammen, da dies einen 400 m mächtigen Mosel-Gletscher bei Remiremont voraussetzen würde, was im Widerspruch zur nahegelegenen Endmoräne von Pouxeux steht. Eine rißeiszeitliche Lokalvereisung kann ebenfalls ausgeschlossen werden, denn die Zusammensetzung der Moräne läßt ebenso wie die petrographische Vielfalt und die Größe der Erratika auf ein umfangreiches Einzugsgebiet zurückschließen.

Von SERET abgesehen, gehen die meisten Autoren von mindestens drei selbständigen Eiszeiten - FLAGEOLLET (1985: 185) hält sogar 4-5 Glaziale für möglich - in den Südvogesen aus. Die im Untersuchungsgebiet ausgewerteten Belege bestätigen die Existenz von mindestens drei Kaltzeiten, wobei die eisbedeckte Fläche von Glazial zu Glazial abnahm. Bei den beiden ersten handelt es sich um das Gebirge überdeckende Kappenvereisungen, die sich zum Rand hin in kurze Talgletscher auflösten.

\section{Schriftenverzeichnis}

Flageollet, J.-C. \& Hameurt, J. (1971): Les accumulations glaciaires de la vallée de la Cleurie (Vosges). - Revue Géogr. de l'Est.: 119-181, 7 Abb., 4 Phot.; Nancy.

Flageollet, J.-C. (1984): Cent cinquante ans de recherches sur le glaciaire vosgien. Histoire des idées et état de la question. - Mélanges offerts à André Journaux: 173-192, Caen. 
HANTKE, R. (1978): Eiszeitalter. Die jüngste Erdgeschichte der Schweiz und ihrer Nachbargebiete, 1: 398-411, 9 Phot., 2 Kt.; Thun (Ott).

HoL, J. B. L. (1940): Een glaciaal dal in de Vogezen: het 'Vallée des lacs' (Vologne-Cleurie-dal). - Tijdschr. K. ned. Aardrijksk. Genoot., 57: 816-838; Amsterdam.

RAHм, G. (1970): Die Vergletscherungen des Schwarzwaldes im Vergleich zu denjenigen der Vogesen. - Alem. Jb. 1966/67, 17: 257-272, 2 Kt.; Bühl-Baden.

SALOME, A. I. (1968): A geomorphological study of the drainage area of the Moselotte and Upper Vologne in the Vosges (France). - Diss.: Univers. Utrecht; $98 \mathrm{~S}$., 17 Abb., 4 Tab., Diagr. u. Phot.; Utrecht.

SERET, G. (1966): Les systèmes glaciaires du bassin de la Moselle et leurs enseignements. - Revue belge de Géographie, 90: 155-577, 77 Abb., 3 Tab., 15 Phot.; Brüssel.

- (1980): La stratigraphie des glaciations dans les vosges du sud. Problèmes de stratigraphie quaternaire en France et dans les pays limitrophes. - Supplt. Bull. A.F.E.Q., 1: 107-109, 1 Abb.; Lyon.

- (1985): Die eiszeitlichen Vergletscherungen der Lothringischen Vogesen und ihre Stratigraphie. DEUQUA-Exkursionsführer II: 15-97, 36 Abb., 3 Tab.; Hannover.

\section{Geologische Karten und Führer}

HAMEURT, J. (1967): Carte géologique des terrains cristallins et cristallophylliens des vosges moyennes lorraines 1: 100000 . - Bull. Serr. Carte géol. Als. Lorr., 20, 2: 117-130; Strasbourg.

Service Geologique National (1978): Carte géologique détaillée de la France au 1:50000, Blatt Gérardmer.

- (1979): Carte géologique détaillée de la France au 1: 50000 , Blatt Remiremont.

vON Eller, J.-P. et al. (1984): Vosges-Alsace. - Guide géologique régional: $182 \mathrm{~S}$., 90 Abb., 7 Tab., 5 Taf.; Paris, 2. Aufl. (Masson).

Manuskript eingegangen 4. 8. 1986. 
